\title{
Improved Direct Routing Approach for Mobile IP Systems
}

\author{
https://doi.org/10.3991/ijim.v13i08.10721
}

\author{
Ali Alshahrani \\ Arab Open University, Riyadh, KSA \\ a.shahrani@arabou.edu.sa
}

\begin{abstract}
The mobile IP communication protocol is a flexible system for mobile connection that is designed to allow the device to be moved between networks while maintaining a permanent IP address. IP datagrams using mobile IP can be routed to a specific mobile station (MS) regardless of its current internet location. However, to facilitate such routable characteristics, the communication with an MS that is visiting a foreign network is intermediate with communication between the home agent (HA) and the foreign agent (FA) which increase the total delay and harms the communication performance, especially when the MS continuously moves from a network to another. Accordingly, to handle the delay issue, this paper proposes a new approach for mobile IP direct routing, based on the "follow-me" principle. The proposed approach depends on sending a new temporary care-of-address $(\mathrm{CoA})$ for the previously visited network as the MS moves to another to keep a reliable tracking-like process with the HA. Accordingly, the agents of all the visited networks play the same principles as the HA role whenever the MS moves from a network to another. The proposed approach influences the delay and the speed of the delivery of internet packets. The simulation showed that the proposed method decreases the delay by up to $50 \%$ compared to the direct routing approach.
\end{abstract}

Keywords-Mobile IP, Routing, Care of address, Home Agent, Foreign Agent

\section{Introduction}

Mobile IP technology has been developed to ensure that a user of any mobile device can move from one network to another while maintaining a permanent IP address [1]. As a location independent, using mobile IP allows the IP datagrams to be routed by identifying each mobile station (MS) with a home address regardless of its current internet location. Accordingly, if the MS is positioned away from the home network, it is associated with a particular address, known as the care-of-address (CoA), which identifies its current location using the endpoint [2]. Generally, mobile IP is responsible for the registration of the MS with the home agent (HA) and routing the datagrams via the tunnel to the MS. The mobile IP usually maintains the TCP connection between the static and the mobile hosts and reduces the effects that are resulted from the mobility of the device; therefore, it helps to keep the TCP connection intact [10]. As 
such, mobile IP is often regarded as a communication protocol that is controlled and standardized by the Internet Engineering Task Force (IETF).

MS is associated with two addresses, the care-of-address $(\mathrm{CoA})$ and the permanent home address. For the implementation of the Mobile IP, two main entities play critical roles in the network connectivity; these are Home Agent (HA) and Foreign Agent (FA). HA stores the information concerning the MSs [3], maintains the entries of the MS location in the directory and acts as a router within the home network of the MS. Accordingly, HA ensures the delivery of datagrams through the tunneling technique to the MS. FA stores the information about all the mobile devices that are visiting the network and advertises the CoA [1]. The FA communicate with the MS to deliver and de-tunnel datagrams, which are then tunneled through the HA by the mobile host. In the absence of FA in the host network, the mobile device has to take over the responsibility of advertising and to address itself. In this case, the FA is usually apprehended as a router that offers a routing service and operates within the network that may be visited by various MSs [4]. This mechanism forms a unique architecture for the mobile IP as illustrated in Figure 1.

Mobility management provides seamless connectivity and session continuity between an MS and the network during its movement. HA is a central entity that tracks the movement of the IP address to keep MS connected and anchoring traffic from source to destination through point-to-point tunnels. The mobility challenge in mobile IP technology is growing with the rapid growth of mobile communication over IP, and the traffic in mobile IP technology will exceed wired communication in terms of the traffic load [5]. According to Cisco [6], the traffic load of the mobile data will be increased by a factor of 11 from 2013 to 2018, and 61\% of the IP traffic will be dedicated to the routable devices. Accordingly, reducing the unnecessary traffic generated by changing the MS location is necessary to improve the performance of the network and reduce the total delay. Generally, the traffic is duplicated by the routing process in the mobile IP, in which both HA and FA intermediate the communication of a visited MS to facilitate its connectivity.

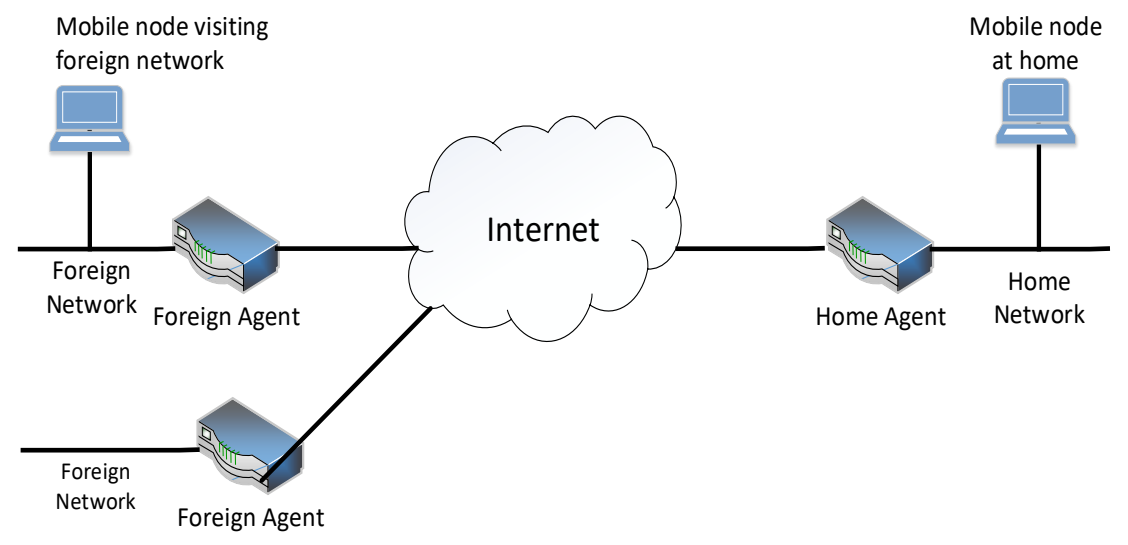

Fig. 1. Mobile IP Network Architecture 
Two approaches are used to facilitate the mobile IP routing process; these are direct and indirect. These routing approaches showed robust features in routing the IP datagrams from a correspondent $(\mathrm{CN})$ and $\mathrm{MS}$, yet, each of which has its scalability limitations with routing via the anchor point. This limitation affects the performance of the network in general in terms of delay and transmission rate. Although the anchorless alternative is not possible due to the architecture of the Mobile IP technology, an enhanced anchor-based approach can be implemented by reliable tracking-like process with the HA in order to reduce the communication overhead while the MH is moving from a network to another.

This paper proposes a new approach, referred to as "follow me" approach that decreases the delay and increase the speed of the data transmission by sending a new temporary IP address (CoA) for the previously visited network as the MS moves from one network to another. The proposed approach keep the HA updated with the MS address by sending a temporary CoA continuously. Although updating the HA with MS routing required extra traffic load, it is trivial compared to the extra traffic load added by both the direct and indirect approach via the anchor point. Accordingly, the proposed approach decreases the load and total delay. Moreover, the proposed approach depends on ease the dependence on the anchor point to save the bandwidth. The detail of the proposed approach will be discussed in the following sections. The rest of the paper is organized as follows: Section 2, presents an overview of the direct and indirect routing approaches. Section 3 discusses the related work on mobility management in mobile IP technology. Section 4 presents the proposed work and discusses the technical details of the "follow me" approach. The experimental simulation results are presented in Section 5. Finally, the conclusion is presented in Section 6.

\section{Mobile IP Routing: Overview}

In the Mobile IP indirect routing approach, the $\mathrm{CN}$ sends a packet addressed by an MS's permanent address to the home network. Accordingly, there is no difference in the routing process in case that the MS is located in its home network or is visiting a foreign network. Thus, mobility is entirely transparent to the $\mathrm{CN}$. In the transmission process, the packet is first forwarded as usual, to the MS's home network. Then, the HA interacts with an FA to trace the mobile station's CoA, as illustrated in Figure 2. The HA also looks out for packet arrival that is addressed to the MS whose home network is that of the HA, but that is currently located in a foreign network. The HA receives these IP packets and then forwards them to a mobile station in a two-step process [7]. First, the packet is forwarded to the FA, using the MS's CoA. Second, the packet is forwarded from the FA to the mobile station [8]. The HA needs to address the packet using the MS's CoA so that the network layer will route the datagram to the external network using the traditional routing algorithms. HA always encapsulates the CN's original datagram with a new larger datagram, which is addressed and delivered to the MS's CoA. The FA, who owns the CoA, will receive and de-capsulate the packet and extract the correspondent's original packet from the larger encapsulating packet $[3,9]$ before forwarding the packet into the MS. 


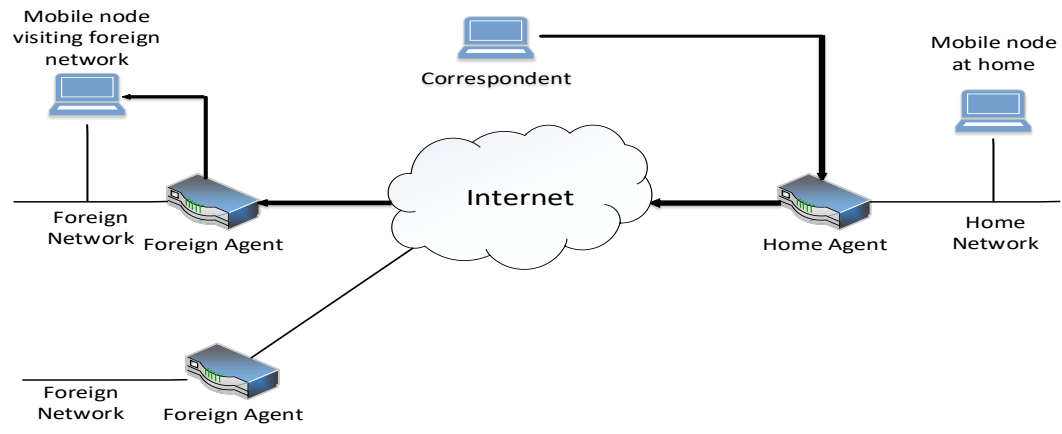

Fig. 2. Mobile IP Indirect Routing

The indirect routing approach suffers from an inefficiency known as the triangle routing problem because the datagrams addressed to the MS must be routed first to the HA and then to the FA. Direct routing of mobile IP is a process in which a correspondent sends the IP packet(s) to an MS without passing through the HA. The route that is adopted by the packet for traveling is called direct route, and the process implemented by such route is called direct routing. In the mobile IP direct routing approach, a CN sends a request to an $\mathrm{HA}$ for the address of the MS. After receiving the permanent mobile address or the current CoA of the requested MS, the $\mathrm{CN}$ directly forward the packet(s) to the network where the MS is currently located, as illustrated in Figure 3 [10].

Accordingly, the direct approach reduces the dependency of the HA in forwarding the transmitted packets to the moving device, yet, the $\mathrm{CN}$ has to request for the address from the MH while transmitting the first packet. While this is small communication overhead, the significant communication overhead results when an MS keep moving from a network to another as packets are addressed to it, leading to another situation that is similar to the indirect approach. A comparison between the direct and indirect approaches based on the responsibility of the involved nodes is given in Table $1[11,12]$.

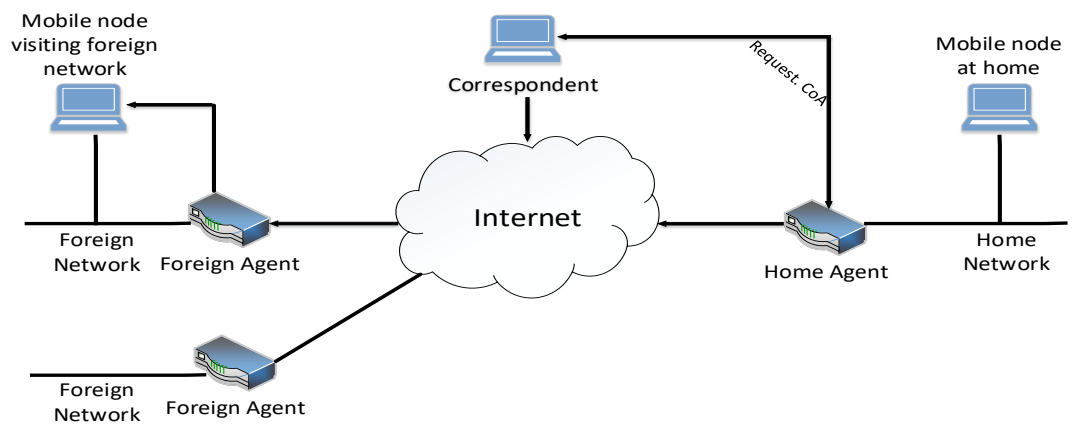

Fig. 3. Mobile IP Direct Routing 
Table 1. Comparison between Direct and Indirect Approach

\begin{tabular}{|l|l|l|}
\hline & \multicolumn{1}{|c|}{ Indirect } & \multicolumn{1}{|c|}{ Direct } \\
\hline$H A$ & $\begin{array}{l}\text { Receives all the transmitted packets and } \\
\text { transfers them to the FA. }\end{array}$ & $\begin{array}{l}\text { Receives the first transmitted packet and trans- } \\
\text { fers it to the FA. Receives and provides the } \\
\text { CoA. }\end{array}$ \\
\hline FA & $\begin{array}{l}\text { Receives all the transmitted packets and } \\
\text { transfers them to the MS. } \\
\text { Traces the CoA of the visiting MS. }\end{array}$ & $\begin{array}{l}\text { Receives the first transmitted packet and trans- } \\
\text { fers it to the FA. Registers CoA to the visiting } \\
\text { MS. }\end{array}$ \\
\hline$M S$ & Registers CoA with the FA & Registers CoA with the FA \\
\hline$C N$ & Sends all the transmitted packets to the HA. & $\begin{array}{l}\text { Sends the first transmitted packet to the HA and } \\
\text { communicates directly with the MS. }\end{array}$ \\
\hline
\end{tabular}

\section{Related Work}

The mobile IP routing approach allows users to move between networks and continue receiving the incoming session requests and support sessions in progress [13]. The previously discussed approaches for routing the mobile IP have disadvantages that have been addressed in the literature. Wong and Leung [14] compared between the performance of the direct and indirect approaches and conclude that "careful design is necessary in order to reduce the signaling and processing overhead for location update." Ho and Akyildiz [15] and Li et al. [16] provided an estimation of cost for the mobility of the mobile nodes across networks. Based on the conclusion of the cost estimation, various approaches for minimizing the communication overhead in mobile IP routing have been proposed. The researches for improving the mobility management have taken different directions, which can be summarized as follows: Estimation nodes' location for cost reduction, decentralization the communication using agents other than the network agent (i.e., HA and FA) for overhead reduction and estimating the closest agent for overhead reduction.

Choi and Tekinay [17] minimize the overhead of the location management process adaptively by predicting the mobility pattern. The adaptive estimation of the mobile location that is moving is only possible if the speed and path information is available and the mobility is taken place at a certain speed on a directed path. Adaptive estimation cannot be used to locate a randomly moving station. Thus, before the location is estimated, the history of the mobile movements are used to determine whether the location can be automatically estimated or not. Accordingly, adaptive estimation eliminates the need for further communication to locate the moving device. Similarly, Taheri and Zomaya [18] proposed an approach based on data clustering algorithm in order to estimate the location of the moving devices. The history of a device movement is used to predict the future movement of the node. The clustering algorithm is used to discover the network topology and automatically determine the routing path.

Zheng et al. [19] proposed an approach that allows the visiting node to use a temporary agent in the visited network. Accordingly, communication is intermediate with dynamic HA (DHA) to reduce the risk of a single point of failure and provide a flexible system. DHA assigns a temporary home address to the visiting node to shorten the distance between the visiting node and the HA, which in turn reduce the handoff latency. 
Yen et al. [20] proposed a Global Dynamic Home Agent Discovery (GDHAD) that allows the visiting node to use the nearest HA through anycast protocol that discovers the closest HA to every node. This approach reduces the cost and delay of registration with the original HA. Cuevas et al. [21] proposed another distributed HA-based approach to reduce cost and latency. The proposed approach is based on peer-to-peer HA discovery. The proposed approach depends on overlay peer-to-peer networks formed by multiple HAs. Each HA has information about the location of the other HAs. An MS sends its current location to the current HA continuously and the current HA lunches a discovery process to find the closest HA to the MH. The current HA sends the MH the IP addresses of the closest HAs, who in turn connect to one of these HAs. Motoyoshi et al. [22] proposed a similar approach for locating the closest HA. The $\mathrm{MH}$ sends a registration request to the current HA, who maintains the path and history information of the MH and the distributed HA. The current HA finds the most suitable HA based on the history maintained and starts the delegation procedure with the selected HA. The current HA sends an acknowledgment to the HA as the delegation completed. Chen et al. [23] propose a mobility management approach to reduce the cost of communication using a Markov process that analyzes the tracking cost of the device mobility. A mobile subscriber (MS) grouping and equivalent quadrangular area are used to reduce the communication overhead with the stations. A summary of the reviewed literature is given in Table 2.

Table 2. Comparison between Mobile IP Routing Enhancement Approaches

\begin{tabular}{|l|l|l|}
\hline \multicolumn{1}{|c|}{ Approach } & \multicolumn{1}{|c|}{ Goal } & \multicolumn{1}{c|}{ Approach Description } \\
\hline $\begin{array}{l}\text { Choi and Tekinay } \\
{[17]}\end{array}$ & $\begin{array}{l}\text { Estimation nodes' location for } \\
\text { cost reduction. }\end{array}$ & $\begin{array}{l}\text { Adaptively predicting the mobility pattern to deter- } \\
\text { mine the location. }\end{array}$ \\
\hline $\begin{array}{l}\text { Taheri and Zoma- } \\
\text { ya [18] }\end{array}$ & $\begin{array}{l}\text { Estimation nodes' location for } \\
\text { cost reduction. }\end{array}$ & $\begin{array}{l}\text { Estimates the location of the moving devices using } \\
\text { network topology analysis. }\end{array}$ \\
\hline Zheng et al. [19] & $\begin{array}{l}\text { Decentralization communication } \\
\text { using agents other than HA. }\end{array}$ & $\begin{array}{l}\text { Allows the visiting node to use a temporary HA in } \\
\text { the visited network. }\end{array}$ \\
\hline Yen et al. [20] & Estimating the closest HA & $\begin{array}{l}\text { Allows the visiting node to use the nearest HA } \\
\text { through anycast protocol. }\end{array}$ \\
\hline Cuevas et al. [21] & Estimating the closest HA & $\begin{array}{l}\text { The proposed approach is based on peer-to-peer HA } \\
\text { discovery in overlay peer-to-peer networks. }\end{array}$ \\
\hline $\begin{array}{l}\text { Motoyoshi et al. } \\
\text { [22] }\end{array}$ & Estimating the closest HA & $\begin{array}{l}\text { HA maintains the path and history information of the } \\
\text { MH and the distributed HA to find the suitable HA. }\end{array}$ \\
\hline Chen et al. [23] & Estimating the closest HA & $\begin{array}{l}\text { Uses a Markov process that analyzes the tracking } \\
\text { cost of the device mobility and finds the best HA. }\end{array}$ \\
\hline
\end{tabular}

Although the existing literature addressed the problem of delay and communication overhead by locating and communicating with the HA of the visited network, the solution to the cost of registration and intermediate communication are still challenging the mobile IP technology. Accordingly, a new approach for mobile IP routing is required to solve this problem, which will be addressed in this paper. 


\section{The Proposed Approach}

A new approach called Follow me is proposed by improving the commonly utilized direct routing approach for mobile IP. The proposed approach depends on sending a new temporary IP address (CoA) for the previously visited network as the MS moves to another network. Besides, the mobile node also registers its new $\mathrm{CoA}$ in its home network to keep a reliable tracking-like process with the home agent (HA).

\subsection{The "Follow me" CoA registration process}

A mobile station (MS) in mobile IP can be moved from a network to another. Accordingly, a mechanism for CoA registration is required with the HA and FA. In follow me approach, when the MS moves from its home network to any foreign network, the foreign agent (FA) of a host network immediately sends a new temporary IP address (CoA) for the visiting MS. The MS then registers its new CoA in its home network. Then, when the MS leaves the visiting network and moves to another, the previous CoA will be discarded, and a new CoA from the current network's FA will be received and registered with the HA and the previously visiting network's HA. Accordingly, if no transmission process is being implemented while the MS moves, a correspondent will ignore the old CoA immediately after receiving the new CoA. However, if a transmission process is implemented while MS moves, the agent of the previously visiting network will receive the packets and transmitted them to the MS based on the recently received CoA. Then, after the internet session between MS and a $\mathrm{CN}$ is terminated using the TCP 3-way handshaking, the $\mathrm{CN}$ will use only the MS permanent IP address for the future connection with the underlying MS. To explain the process of CoA registration for the proposed system, an example of an autonomous network system is illustrated in Figure 4.

As given in Figure 4, the MS left the home network and moved towards a foreign network $\left(\mathrm{FN}_{1}\right)$. After a while, the MS moves to $\mathrm{FN}_{2}, \mathrm{FN}_{3}, \mathrm{FN}_{7}$ and finally to $\mathrm{FN}_{\mathrm{n}}$. Each time it moves, the MS receives a temporary address, which is then registered with the home network of the MS and is sent directly to the previous FN. Assume that the MS receives $\mathrm{Co}_{1}$ from $\mathrm{FN}_{1}$ for $\mathrm{T}_{1}$ period when it moves to the first foreign network, where $T_{1}$ is the time the MS will remain in the network associated with $\mathrm{FN}_{1}$. A session was in progress with a particular $\mathrm{CN}$ when MS moves from $\mathrm{HN}$ to $\mathrm{FN}_{1}$. The MS sends its new temporary address $\mathrm{CoA}_{1}$ to the $\mathrm{HA}$ and to the $\mathrm{CN}$ to ensure the internet session remains in use. Then, when the MS leaves $F N_{l}$ and moves towards $F N_{2}$, it will lose $\mathrm{CoA}_{1}$ and receive a new temporary IP address $\mathrm{CoA}_{2}$ from $F N_{2}$. The MS will send $\mathrm{CoA}_{2}$ to the $F N_{l}$ and the HA. During the mobility of MS, when it will move through $F N 3$, the same process of CoA registration will be repeated using $\mathrm{CoA}_{3}$ from $F N_{3}$ then $\mathrm{CoA}_{4}$ from $F N_{7}$ followed by $\mathrm{CoA}_{5}$ from $F N_{n}$. Accordingly, the MS always sends the new CoA to the previously visited network to notify it about its current address. Thus, if a packet is being transmitted while the MS is moving, the notified FA will direct the packet into the address that is sent by the moving MS. In such a way, re-transmission is avoided in all cases, which reduce the delay and en- 
hance performance. The process of registering and distribution of the MS for the example that is given in Figure 4 is illustrated in Figure 5.

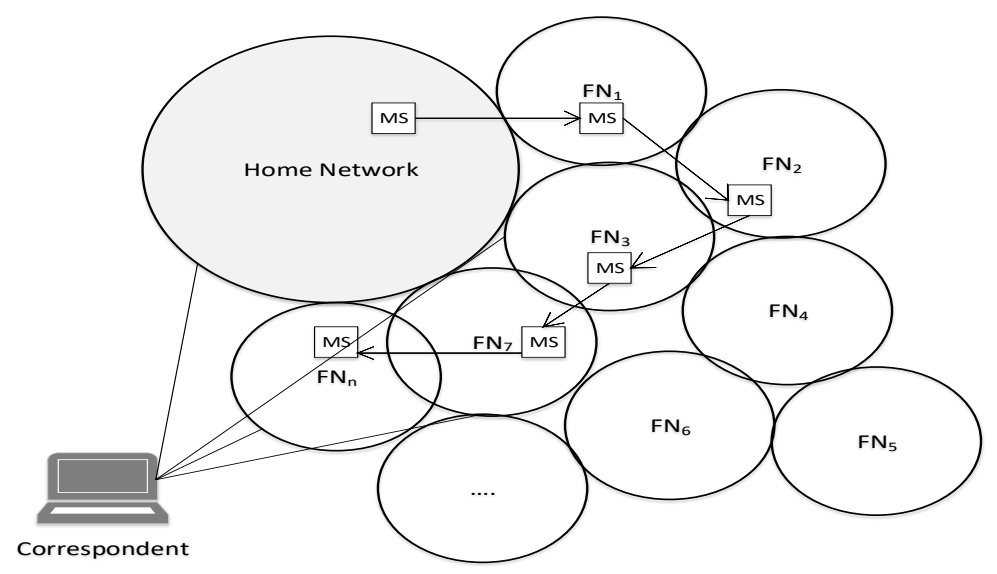

Fig. 4. Example of a Mobile station Mobility process

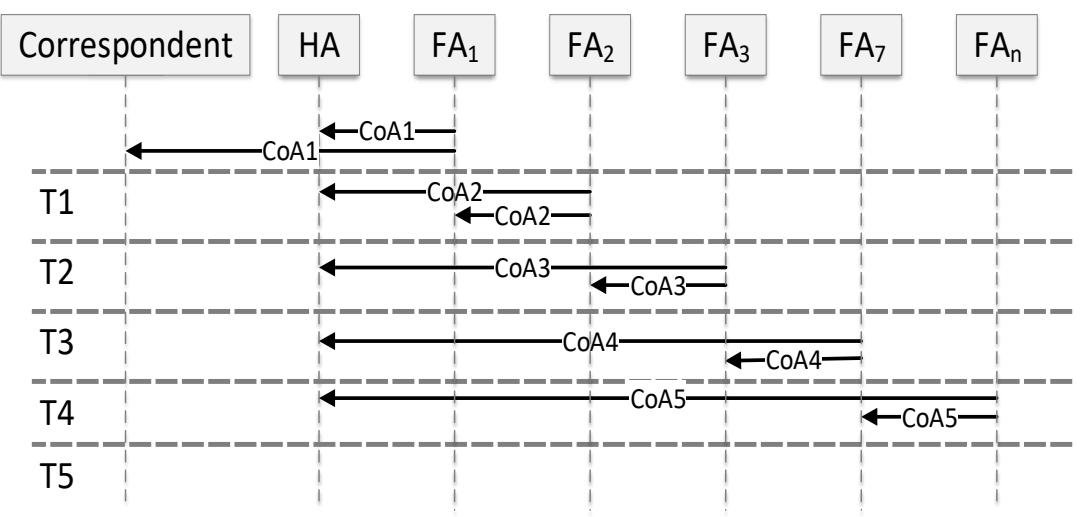

Fig. 5. Mobile IP "follow me" Direct Routing CoA Registration

\subsection{The "Follow me" packet delivery mechanism}

Packet delivery mechanism in the proposed follow me approach is implemented based on the location of the MS. The first scenario occurs when the MS is located within the home network. In this case, the $\mathrm{CN}$ will directly send a packet to the HA using the traditional internet routing techniques, taking into consideration the fact that the destination IP address of the packet is the mobile's permanent address. As the mobile is located within the home network scope, the packets will be directly forwarded to the desired MS. The MS then receives the packet and gives the required 
responses as requested. While when the MS is located within a foreign network, the MS will have a temporary address that was obtained from the foreign network and registered with the network agent(s). The $\mathrm{CN}$ transmits a packet to the MS, based on the CoA according to the following scenarios:

- The CN directly sends the first IP packet to the HA who will encapsulate the packet and forward it to the last visited network. After that, HA sends a particular service packet that contains the $\mathrm{CoA}$ to the $\mathrm{CN}$ that enable $\mathrm{CN}$ to send packets directly to MS without referring back to HA.

- The CN sends a request to the HA to inquire whether the current IP address of the MS is MA or CoA. After receiving the address, the corresponding network forwards the packet directly to the MS that is located in a visiting network.

- The $\mathrm{CN}$ sends a request to the HA to inquire whether the current IP address of the MS is MA or CoA. After receiving the address, the corresponding network forwards the first packet directly to the MS that is located in a visiting network.

The most critical scenario occurs when $\mathrm{CN}$ is transmission packets while the MS is moving to another network while. The previous FA forwards the packet from $\mathrm{CN}$ to the first network, and the previous FN will inform the $\mathrm{CN}$ about the new CoA for the MS. Accordingly, using the proposed packet delivery mechanism, the end to end delay will be enhanced, and the reliability of the transmission will be improved through the prevention of packet loss that may occur due to the movement of the mobile station in the visiting networks. The proposed mechanism minimizes the communication with the HA, as the correspondent refers to the HA only if the transmission is failed. Moreover, the FA will also have less overhead as the MS is responsible for acquiring the CoA once.

Example of the packet delivery mechanism in the proposed follow me approach compared to the traditional direct approach is illustrated in Figure 6. Assume that the $\mathrm{CN}$ sends the first packet directly to the MS that is located in R12. The MS moves to $\mathrm{R} 9$, and the packet from $\mathrm{CN}$ to $\mathrm{R} 12$ is not delivered. In the traditional direct approach, the $\mathrm{CN}$ will contact the HA for new CoA for the moving device then the packet will be sent again from the $\mathrm{CN}$ to R9. In this case, the total delay will be the end-to-end delay plus the encapsulation and de-capsulation process time for the two transmission attempts. While in the proposed follow me approach, R12 will inform for the $\mathrm{CN}$ about the new CoA for the moving device and the packet will be forwarded from R12 to R9. Accordingly, the time consuming double transmission will be eliminated using the follow me approach. 

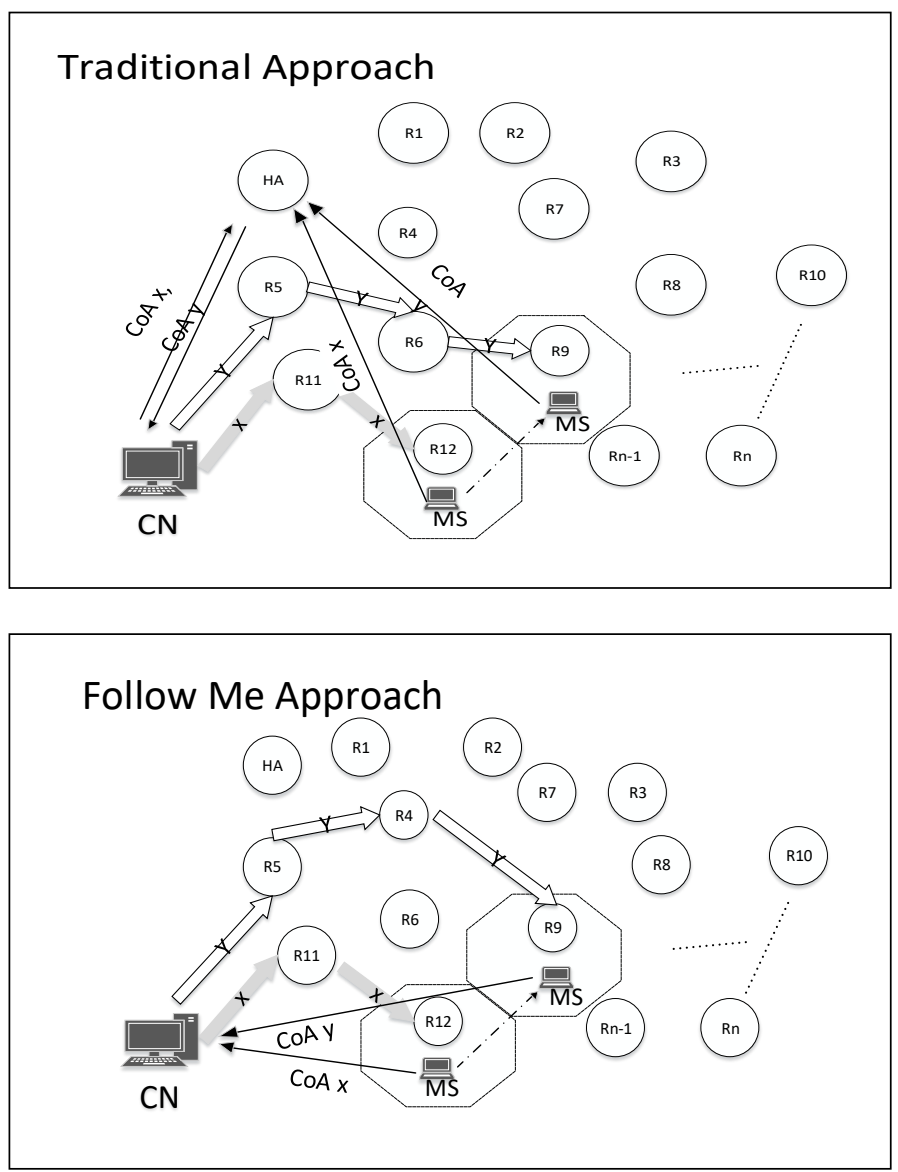

Fig. 6. Packet Delivery Process in Follow me and the Traditional Direct Routing

\section{Comparison, Simulation, and Results}

Numerical analysis and simulation are conducted to evaluate the performance of the proposed approach. Accordingly, six scenarios are implemented for the mobility of the devices, and the total delay at each scenario is calculated. A summary of the scenarios is given in Table 3 .

Accordingly, there are three different delays in any of these scenarios; these are transmission delay (Ds), Encapsulation and de-encapsulation delay (Den) and CoA transmission delay (DCoA). These different forms of delay based on the depicted scenario will be evaluated using numerical simulation and mobile simulation. In the numerical analysis, without loss of generality, we assume that the transmission delay is identical regardless of the identity of the sender and the receiver nodes. Accordingly, we also assume that the transmission delay and the CoA transmission delay are 
identical. Accordingly, the delay of each of the depicted scenario is calculated as given in Table 4.

Table 3. Comparison between the Direct Approach and Follow me Approach

\begin{tabular}{|c|c|c|c|}
\hline Scenario & & Direct Approach & Proposed Approach \\
\hline \multirow{2}{*}{$\begin{array}{l}\text { Located Node in } \\
\text { the Home Net- } \\
\text { work }\end{array}$} & First Packet & \multirow{2}{*}{\multicolumn{2}{|c|}{$\begin{array}{l}\text { S1: Total delay is equal to the transmission delay as the packet is sent } \\
\text { directly. }\end{array}$}} \\
\hline & Other Packets & & \\
\hline \multirow[b]{2}{*}{$\begin{array}{l}\text { Located Node in a } \\
\text { Foreign Network }\end{array}$} & First Packet & \multicolumn{2}{|c|}{$\begin{array}{l}\text { S2: Total delay is equal to the transmission delay from } \mathrm{CN} \text { to } \mathrm{HA} \text { and } \\
\text { HA to MS plus the encapsulation delay. }\end{array}$} \\
\hline & Other Packets & \multicolumn{2}{|c|}{$\begin{array}{l}\text { S3: Total delay is equal to delay of sending a particular service packet } \\
\text { that contains the CoA to the CN after the first packet and the transmis- } \\
\text { sion delay from } \mathrm{CN} \text { to MS plus the encapsulation delay. }\end{array}$} \\
\hline \multirow[t]{2}{*}{$\begin{array}{l}\text { Relocated Visiting } \\
\text { Node }\end{array}$} & First Packet & $\begin{array}{l}\text { S4: Total delay is equal to the } \\
\text { transmission delay from CN to } \\
\text { HA and HA to departed network } \\
\text { and the encapsulation delay. } \\
\text { Besides, the delay of encapsula- } \\
\text { tion and re-transmission delay n- } \\
\text { times, to n-networks that are } \\
\text { visited by the MS while the packet } \\
\text { is transmitted. }\end{array}$ & $\begin{array}{l}\text { S5: Total delay is equal to the } \\
\text { transmission delay from } \mathrm{CN} \text { to HA } \\
\text { and HA to departed network and the } \\
\text { encapsulation delay. Besides, the } \\
\text { delay of re-transmission delay } \\
\text { between n-times, to n-networks that } \\
\text { are visited by the MS while the } \\
\text { packet is transmitted. }\end{array}$ \\
\hline & Other Packets & \multicolumn{2}{|c|}{$\begin{array}{l}\text { S6: Total delay is equal to delay of sending a particular service packet } \\
\text { that contains the CoA to the CN after the first packet and the transmis- } \\
\text { sion delay from CN to MS plus the encapsulation delay. }\end{array}$} \\
\hline
\end{tabular}

Table 4. Representation of the Total Delay in the Direct Approach and Follow Me Approach

\begin{tabular}{|c|l|c|}
\hline Scenario & \multicolumn{1}{|c|}{ Equation } & Unity Measure \\
\hline$S 1$ & $D_{\text {total }}=D s$ & 1 \\
\hline$S 2$ & $D_{\text {total }}=D s_{C N-t o-H A}+D s_{H A-t o-F A}+D_{e n}$ & $\mathbf{3}$ \\
\hline$S 3$ & $D_{\text {total }}=D s_{C N-t o-H A}+D s_{H A-t o-F A}+D_{e n}+D_{C O A}$ & $\mathbf{4}$ \\
\hline$S 4$ & $D_{\text {total }}=D s_{C N-t o-H A}+\left(\sum_{i=1}^{n} D s_{i}\right)+\left(\sum_{i=1}^{n} D_{c o A} i\right)+D_{e n}$ & $\mathbf{2 n}+\mathbf{2}$ \\
\hline$S 5$ & $D_{4}=D s_{C N-t o-H A}+\sum_{i=1}^{n} D s_{i}+D_{C o A}$ & $\mathbf{n}+\mathbf{2}$ \\
\hline$S 6$ & $D_{\text {total }}=D s_{C N-t o-H A}+D s_{H A-t o-F A}+D_{e n}+D_{C O A}$ & $\mathbf{4}$ \\
\hline
\end{tabular}

Accordingly, the apparent delay occurs when the MS is moving continuously from a network to another forming the first-packet scenario repeatedly. Accordingly, if the relocating of the MS occurred with each packet transmission with a probability of 1 , the direct approach delay will be almost double the delay in the follow me approach. The variation in delay between the two approaches decreases as the probability is decreased till the variation reach zero with moving probability of 0 .

A mobile IP system was simulated using Java with four types of agents; these are HA, FA, MS, and CN. A set of functionalities with various frame types were developed to facilitate communication within the simulated environment. The mobile node can send a message, register with HA and deregister with FA. CN listen to incoming 
messages are reply accordingly. The HA initiate a communication with the $\mathrm{CN}$ and with the FA to send messages to MS. The HA inform $\mathrm{CN}$ about the newly registered nodes and communicate messages with nodes and FA. The FA register MS nodes and communicate with CN. According to the simulated system, the direct approach and the follow-me approach were simulated based on different relocated probability which creates different scenarios according to the discussion above with different packet numbers and the total delay in a millisecond was captured and compared as illustrated in Figure 7.

As the results illustrate, the total delay of the follow me approach is less than the delay of the direct approach. The resulting gap is increasing when the relocating probability increase, in which the number of relocating of MS occurred more often. In the probability of 1.0, the total delay of the direct approach is almost double for the follow me approach. As noted in the results, the total delay variations are increased among the compared methods as the number of communicated packets increases, which clearly illustrates the benefits of using the proposed approach in reducing the total delay in real communication, in which the number of transmitted packets are substantially huge.

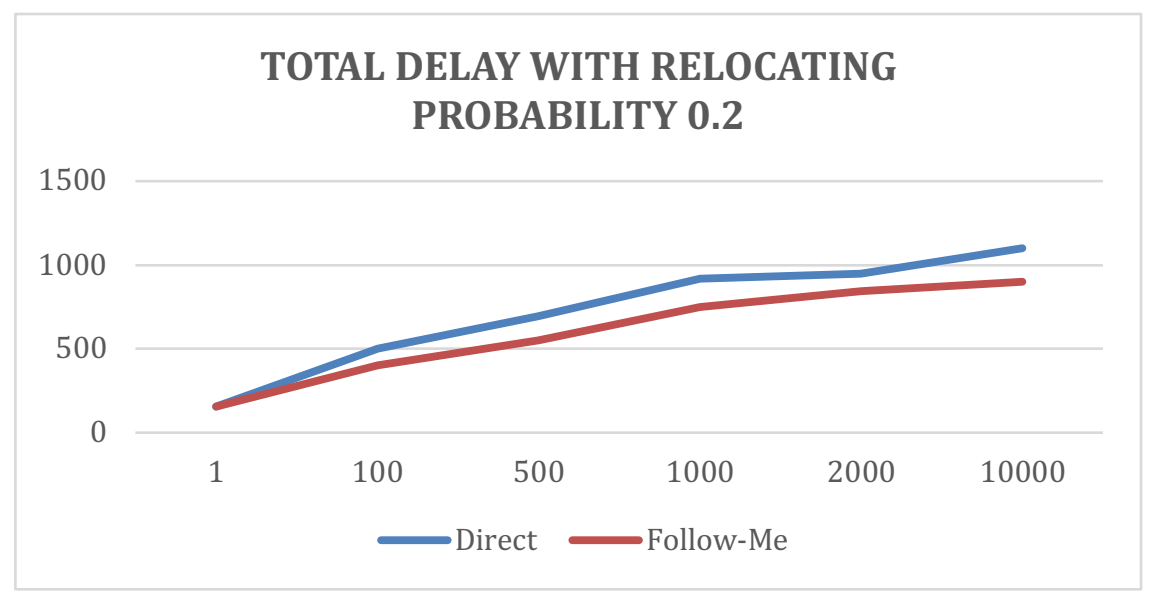



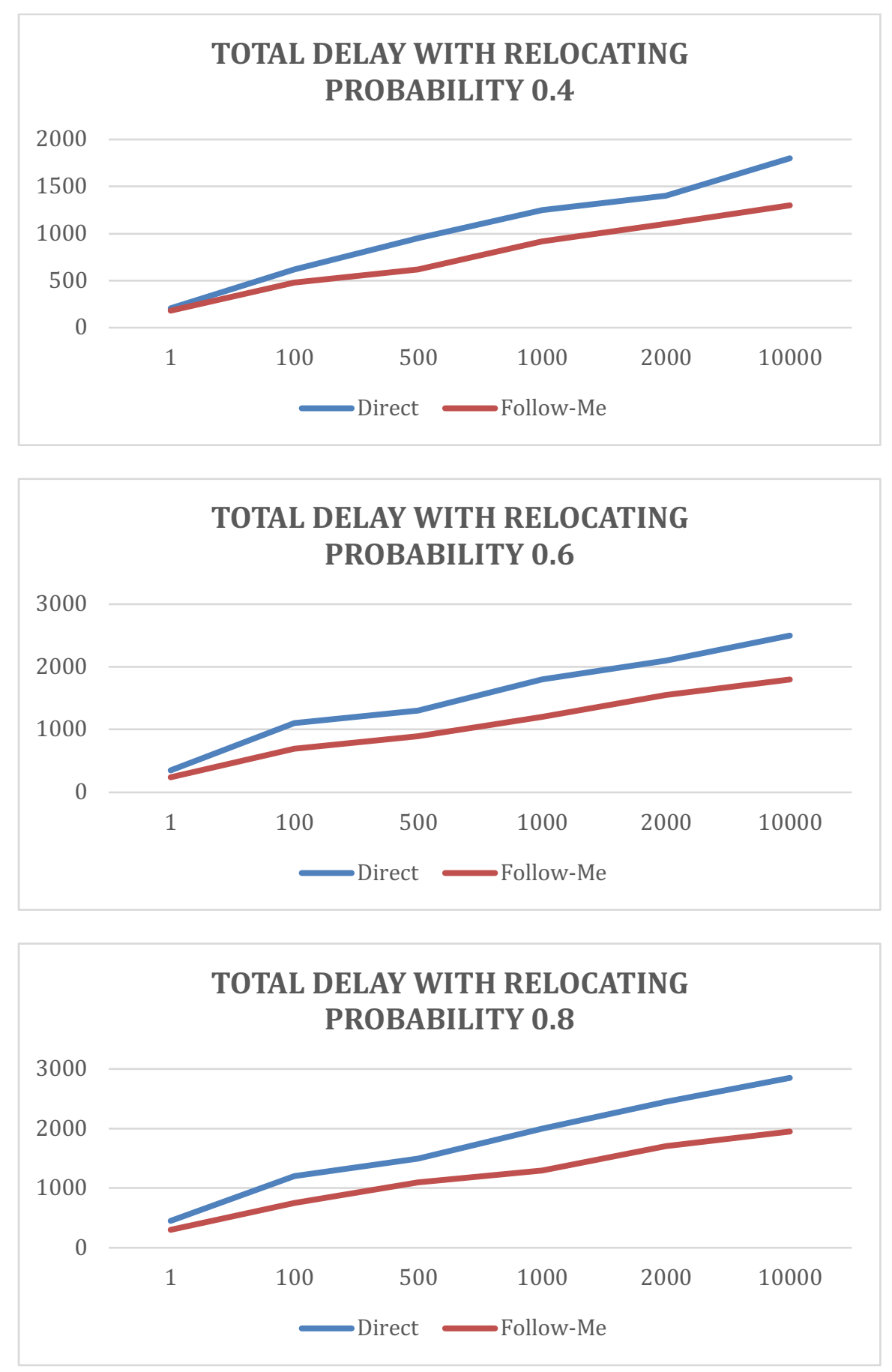


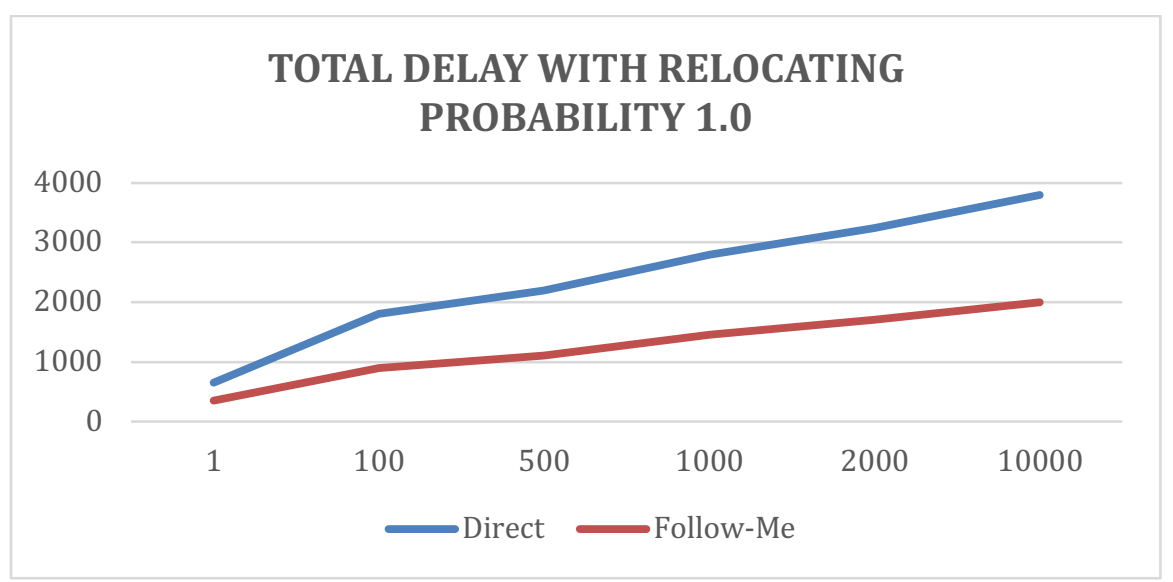

Fig. 7. The total Delay of the Direct Approach and Follow me Approach with different Relocation Probability and Packet Number

\section{Conclusion}

The mobile IP direct routing approach has become a popular method for mobile routing. However, it suffers from the problem of re-transmission as the mobile agent relocated from that network to another. Accordingly, in this paper, a new approach, which is referred to as "follow me", is proposed to manage the direct routing of mobile IP. The proposed method depends on informing and allowing the agent of the previously visiting network to transmitted packets to the MS based on the received CoA. Accordingly, the proposed approach minimizes the communication with the $\mathrm{HA}$, as the correspondent refers to the HA only if the transmission is failed. Moreover, the FA will also have less overhead as the MS is responsible for acquiring the CoA once. The simulation results showed that the total delay could be reduced up to the half in the case with a probability of mobile relocating of one.

\section{$7 \quad$ Acknowledgement}

This work was supported by the Arab Open University, KSA.

\section{References}

[1] Opos, J.M., et al. A performance analysis of indirect routing. in 2007 IEEE International Parallel and Distributed Processing Symposium. 2007. IEEE.

[2] Mohammadpour, M., H. Parvin, and A. Chamkoori, Improvement of Routing Operation Based on Learning with Using Smart Local and Global Agents and with the Help of the Ant Colony Algorithm. Journal of Advances in Computer Research, 2017. 8(4): p. 107119. 
[3] Perkins, C.E., Mobile networking through mobile IP. IEEE Internet computing, 1998. 2(1): p. 58-69. https://doi.org/10.1109/4236.656077

[4] Gohil, A., H. Modi, and S.K. Patel. 5G technology of mobile communication: A survey. in 2013 international conference on intelligent systems and signal processing (ISSP). 2013. IEEE. https://doi.org/10.1109/issp.2013.6526920

[5] Xylomenos, G., et al., Caching and mobility support in a publish-subscribe internet architecture. IEEE Communications Magazine, 2012. 50(7): p. 52-58. https://doi.org/10.1109/ mcom.2012.6231279

[6] Cisco, I., Cisco visual networking index: Forecast and methodology, 2013-2018. CISCO White paper, 2013. 2018.

[7] Clausen, T., U. Herberg, and J. Yi, Security Threats to the Optimized Link State Routing Protocol Version 2 (OLSRv2). 2017. https://doi.org/10.17487/rfc8116

[8] Magnano, A., X. Fei, and A. Boukerche. Predictive mobile IP handover for vehicular networks. in 2015 IEEE 40th Conference on Local Computer Networks (LCN). 2015. IEEE. https://doi.org/10.1109/lcn.2015.7366328

[9] Kurose, J. and K. Ross, Computer networks: A top down approach featuring the internet. Peorsoim Addison Wesley, 2010.

[10] Li, C., et al. Using indirect routing to recover from network traffic scheduling estimation error. in 2017 ACM/IEEE Symposium on Architectures for Networking and Communications Systems (ANCS). 2017. IEEE. https://doi.org/10.1109/ancs.2017.12

[11] Anastasiades, C., T. Braun, and V.A. Siris, Information-centric networking in mobile and opportunistic networks, in Wireless Networking for Moving Objects. 2014, Springer. p. 14-30. https://doi.org/10.1007/978-3-319-10834-6_2

[12] Lee, J. and S. Pack, Mobility management in future wireless networks: Past, present, and future. IEEE WCNC Tutorial, 2013

[13] Al-Surmi, I., M. Othman, and B.M. Ali, Mobility management for IP-based next generation mobile networks: Review, challenge and perspective. Journal of Network and computer Applications, 2012. 35(1): p. 295-315. https://doi.org/10.1016/j.jnca.2011.09.001

[14] Wong, V.-S. and V.C. Leung, Location management for next-generation personal communications networks. IEEE network, 2000. 14(5): p. 18-24. https://doi.org/10.1109/65.871 $\underline{336}$

[15] Ho, J.S. and I.F. Akyildiz. A dynamic mobility tracking policy for wireless personal communications networks. in Proceedings of GLOBECOM'95. 1995. IEEE. https://doi.org/10. $\underline{1109 / \text { glocom. } 1995.500211}$

[16] Li, J., H. Kameda, and K. Li, Optimal dynamic mobility management for PCS networks. IEEE/ACM Transactions on Networking, 2000. 8(3): p. 319-327. https://doi.org/10.1109 /90.851978

[17] Choi, W.-J. and S. Tekinay. An adaptive location registration scheme with dynamic mobility classification. in 2002 IEEE International Conference on Communications. Conference Proceedings. ICC 2002 (Cat. No. 02CH37333). 2002. IEEE. https://doi.org/10.1109/icc.2 002.996892

[18] Taheri, J. and A.Y. Zomaya, Clustering techniques for dynamic location management in mobile computing. Journal of Parallel and Distributed Computing, 2007. 67(4): p. 430447. https://doi.org/10.1016/j.jpdc.2006.12.005

[19] Zheng, R., et al., A case for mobility support with temporary home agents. ACM SIGMOBILE Mobile Computing and Communications Review, 2002. 6(1): p. 32-46. https://doi.org/10.1145/511226.511229 
[20] Yen, Y.-S., et al., Global dynamic home agent discovery on mobile IPv6, in 2nd International Conferenceon Mobile Technology, Applications and Systems. 2005. https://doi.org/ $10.1109 / \mathrm{mtas} .2005 .207129$

[21] Cuevas, R., et al. P2P based architecture for global home agent dynamic discovery in IP mobility. in 2007 IEEE 65th Vehicular Technology Conference-VTC2007-Spring. 2007. IEEE. https://doi.org/10.1109/vetecs.2007.194

[22] Motoyoshi, G., K. Leibnitz, and M. Murata, Proposal and evaluation of a functiondistributed mobility architecture for the future internet. IEICE transactions on communications, 2011. 94(7): p. 1952-1963. https://doi.org/10.1587/transcom.e94.b.1952

[23] Chen, K.-T., S.-L. Su, and R.-F. Chang, Design and analysis of dynamic mobility tracking in wireless personal communication networks. IEEE Transactions on Vehicular Technology, 2002. 51(3): p. 486-497. https://doi.org/10.1109/tvt.2002.1002498

\section{$9 \quad$ Author}

Ali Alshahrani is an assistant professor in ITC department, Arab Open University - KSA. He received his B.Cs degree in Information Technology and Computing, in 2008. His M.Sc. and Ph.D. from University of Essex, UK in Computer science, in 2015. His research interests include network security, image processing, e-learning and mobile systems.

Article submitted 2019-03-22. Resubmitted 2019-05-08. Final acceptance 2019-05-10. Final version published as submitted by the authors. 